Christine Hine (2010). Book Review: NALITA JAMES and HUGH BUSHER, Online Interviewing. London: Sage, 2009.161 pp. ISBN 9781412945318 (hb) ISBN 9791412945325 (pbk). Qualitative Research, 10: 502 DOI: $10.1177 / 14687941100100040606$. The online version of this article can be found at: http://qrj.sagepub.com/content/10/4/502.citation

When teaching online research methods I often begin by outlining a some- what artificial distinction between research which happens to use the Internet as its medium and research which is intrinsically about the Internet as a medium. Nalita James and Hugh Busher's introduction to the challenges and opportunities of online interviewing shows just how misguided it would be to stick with that distinction. Their text explains that online interviewing is not simply a technique that can be learned by applying rules. By referencing a wide range of sociological work focusing on the Internet and beyond, James and Busher show that the Internet is a complex and highly situated phenomenon that cannot simply be 'used' by an interviewer. Instead, the online inter-viewer needs to pay careful attention to the temporal and spatial dynamics created in and through Internet use, and can artfully use online interactions to craft research relationships and contexts appropriate to a particular project. In the process some complex epistemological, ethical and practical issues arise.

The book is divided into ten chapters which move from a discussion of the basic scenarios of online interviewing to cross-cutting issues of ethics (Chapter 5), concerns about credibility and authenticity of both interviewer and interviewee (Chapter 6), and opportunities for the creation and exploration of online communities for research purposes (Chapter 8). The topic of power relations between interviewer and interviewees recurs throughout the discussion of online interviewing, but is particularly the focus of Chapter 7. While much of the early hype about the Internet stressed the equalization of power relations, James and Busher show in some depth that the situation is far more complex and that the emergent power relations in an online interview can be different from the face-to-face situation but still far from equal. A very useful chapter discusses the curation of data from online interviews: although here I would like to have seen a little more detail on various technical alternatives to accompany the broader discussion of principles. 
Both James and Busher have practical experience of using the Internet to conduct qualitative interviews in their work on academic and educational workplaces. These experiences, particularly James' extended interviews with academics about their workplace learning, are used throughout the book to illustrate choices that researchers need to make and some of the effective strategies to employ. Each chapter contains examples in text boxes and ends with bullet-pointed practical tips and suggestions for further reading. The examples encourage the reader to see the relevance of some of the broader theoretical discussions. It would have been useful to include a slightly wider range of examples using different approaches to interviewing, and more transcripts of actual online interview data to allow the reader to visualise the impact of different choices.

The book covers a wide range of literature on Internet relationships, identities and social formations, and ranges beyond the Internet per se into the work of Giddens, Derrida and Bourdieu. This ambition to cover the territory does, however, create some difficulties in the telling. The book moves quickly from reference to reference, often taking observations about the nature of the medium out of the context of the original research which generated it. This does distract from doing justice to any one perspective, and indeed, contradictory positions on the nature of online interactions are mentioned without the conflicts between them being addressed. A novice online interviewer would gain many snippets that could inform a research approach, but they would be hard pushed to construct a coherent overview of sociological perspectives on the Internet. That is not, of course, James and Busher's goal, but it is a shame to lose the nuances when the book is otherwise so comprehensive.

Within the scope of this text focusing on the online interview Busher and James can only briefly explore the whole question of what an interview can and cannot achieve, epistemologically speaking and how, for example, a collaborative feminist interview might differ from a more empiricist approach to the interview as a tool for collection of data about the world. This question, how- ever, runs beneath the surface of their text. The book is therefore probably less appropriate for a beginner lacking a background in methodological debates about the interview and the nature of the data that it generates. A prior grasp of 
this debate, and an existing commitment to a particular style of qualitative interviewing, would greatly add to the impact of the issues that Busher and James discuss. The book is therefore probably most appropriately read by researchers already experienced in practical and epistemological aspects of face-to-face qualitative interviewing. For such a reader, the issues covered by James and Busher will raise many interesting challenges, and prompt a deeper reflection on the processes of online interviewing which could impact back on their understanding of face-to-face interviewing as well.

\section{Christine Hine}

University of Surrey 